\title{
On Neutrino in Deformed Relativity
}

\section{Lukasz Andrzej Glinka*}

Member of the American Association of International Researchers. New York, USA

\section{Editorial}

Glinka [1] has considered the algebraic deformation of Special Relativity, which led to the equation

$$
\left(\gamma^{\mu} \hat{p} \mu+m c^{2}+\sqrt{\alpha} \frac{c}{\hbar} \ell \gamma^{5} \hat{p}^{2}\right) \psi=0
$$

Which can be easily transformed into the pair of Dirac equations

$$
\left(\gamma^{\mu} \hat{p} \mu-M \pm c^{2}\right) \psi=0,
$$

Where $M \pm$ are the emerging mass matrices, which can be written in the form

$$
\begin{aligned}
& M \pm=\mu \stackrel{ \pm}{R} \frac{1+\gamma^{5}}{2}+\mu \frac{ \pm 1-\gamma^{5}}{2}, \\
& \mu \stackrel{ \pm}{R}=-\frac{1}{c^{2}}\left(\frac{\varepsilon}{2} \pm \sqrt{\varepsilon^{2}-4 \varepsilon m c^{2}-4 E^{2}}\right), \\
& \mu \stackrel{ \pm}{L}=\frac{1}{c^{2}}\left(\frac{\varepsilon}{2} \pm \sqrt{\varepsilon^{2}-4 \varepsilon m c^{2}-4 E^{2}}\right),
\end{aligned}
$$

Where $\mu \stackrel{ \pm}{R L}$ reals and the energy parameter are

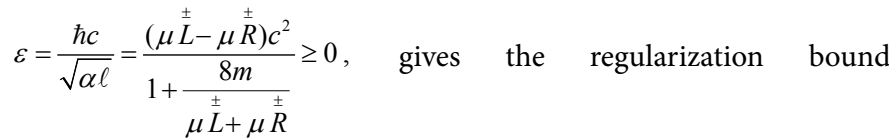
$-\frac{\varepsilon}{2} \leq E \leq \frac{\varepsilon}{2}$, where as the squared-mass difference is $\Delta \mu_{L R}^{2}=\left(\mu_{L}^{ \pm}\right)^{2}-\left(\mu_{R}^{ \pm}\right)^{2}=32 \pi \frac{\ell p}{\ell} M_{p}^{2}$.

Making use of the right- and left-handed chiral Weyl fields

$$
\psi_{R}=\frac{1+\gamma^{5}}{2} \psi, \psi_{L}=\frac{1-\gamma^{5}}{2} \psi
$$

Where solves (2), one gets the system of two massive Weyl equations

$$
\left(\gamma^{\mu} \hat{p}_{\mu}+\mu^{ \pm} c^{2}\right)\left[\begin{array}{l}
\psi_{R}^{ \pm} \\
\psi_{L}^{ \pm}
\end{array}\right]=0, \mu^{ \pm}=\left[\begin{array}{cc}
\mu_{R}^{ \pm} & 0 \\
0 & \mu_{L}^{ \pm}
\end{array}\right]
$$

Which can be rewritten as the two-component Schrodinger equation

$$
i \hbar \partial_{0}\left[\left(\begin{array}{l}
\psi_{R}^{ \pm}(x, t) \\
\psi_{L}^{ \pm}(x, t)
\end{array}\right)=-\gamma^{0}\left(i \hbar c \gamma^{i} \partial_{i}+\left[\begin{array}{cc}
\mu_{R}^{ \pm} c^{2} & 0 \\
0 & \mu_{L}^{ \pm} c^{2}
\end{array}\right]\right)\left(\begin{array}{l}
\psi_{R}^{ \pm}(x, t) \\
\psi_{L}^{ \pm}(x, t)
\end{array}\right) .\right.
$$

For the initial momentum Eigen states $\psi_{R, L}^{ \pm}\left(x, t_{0}\right)$

$$
i \hbar \sigma^{i} \partial_{i} \psi_{R, L}^{ \pm}\left(x, t_{0}\right)=p_{R, L}^{ \pm 0} \psi_{R, L}^{ \pm}\left(x, t_{0}\right),
$$

In the Dirac basis, one obtains

$$
\begin{aligned}
& \left(\psi_{R}^{ \pm}\right)^{D}(x, t)=\left\{\left[\cos \left[\frac{t-t_{0}}{\hbar} E^{D}\left(p_{R}^{ \pm 0}\right)\right]-\right.\right. \\
& \left.-i \mu_{ \pm}^{D} c^{2} \frac{\sin \left[\frac{t-t_{0}}{\hbar} E^{D}\left(p_{R}^{ \pm 0}\right)\right]}{E^{D}\left(p_{R}^{ \pm 0}\right)}\right] \exp \left\{-\frac{i}{\hbar} p_{R}^{ \pm 0}\left(x-x_{0}\right)_{i} \sigma^{i}\right\}\left(\psi_{R}^{ \pm}\right)^{D}\left(x_{0}, t_{0}\right)- \\
& \left.-i p_{L}^{ \pm 0} c \frac{\sin \left[\frac{t-t_{0}}{\hbar} E^{D}\left(p_{L}^{ \pm 0}\right)\right]}{E^{D}\left(p_{L}^{ \pm 0}\right)} \exp \left\{-\frac{i}{\hbar} p_{L}^{ \pm 0}\left(x-x_{0}\right)_{i} \sigma^{i}\right\}\left(\psi_{L}^{ \pm}\right)^{D}\left(x_{0}, t_{0}\right)\right\} \times \\
& \times \exp \left\{-i \frac{\left(\mu_{R}^{ \pm}-\mu_{L}^{ \pm}\right) c^{2}}{2 \hbar}\left(t-t_{0}\right)\right\},
\end{aligned}
$$

And

$$
\begin{aligned}
& \left(\psi_{L}^{ \pm}\right)^{D}(x, t)=\left\{\left[\cos \left[\frac{t-t_{0}}{\hbar} E^{D}\left(p_{L}^{ \pm 0}\right)\right]+\right.\right. \\
& \left.+i \mu_{ \pm}^{D} c^{2} \frac{\sin \left[\frac{t-t_{0}}{\hbar} E^{D}\left(p_{L}^{ \pm 0}\right)\right]}{E^{D}\left(p_{L}^{ \pm 0}\right)}\right] \exp \left\{-\frac{i}{\hbar} p_{L}^{ \pm 0}\left(x-x_{0}\right)_{i} \sigma^{i}\right\}\left(\psi_{L}^{ \pm}\right)^{D}\left(x_{0}, t_{0}\right)- \\
& \left.-i p_{R}^{ \pm 0} c \frac{\sin \left[\frac{t-t_{0}}{\hbar} E^{D}\left(p_{R}^{ \pm 0}\right)\right]}{E^{D}\left(p_{R}^{ \pm 0}\right)} \exp \left\{-\frac{i}{\hbar} p_{R}^{ \pm 0}\left(x-x_{0}\right)_{i} \sigma^{i}\right\}\left(\psi_{R}^{ \pm}\right)^{D}\left(x_{0}, t_{0}\right)\right\} \times \\
& \times \exp \left\{-i \frac{\left(\mu_{R}^{ \pm}-\mu_{L}^{ \pm}\right) c^{2}}{2 \hbar}\left(t-t_{0}\right)\right\},
\end{aligned}
$$

Where $E^{D}\left(p_{R}^{ \pm 0}\right)=c^{2} \sqrt{\left(\frac{\mu_{R}^{ \pm}+\mu_{L}^{ \pm}}{2}\right)^{2}+\left(\frac{p_{R}^{ \pm 0}}{c}\right)^{2}}$, while in the Weyl basis $\left(\psi_{R}^{ \pm}\right)^{W}(x, t)=\left\{\cos \left[\frac{t-t_{0}}{\hbar} E^{W}\left(p_{R}^{ \pm 0}\right)\right]-\right.$ $\left.-i p_{R}^{ \pm 0} c \frac{\sin \left[\frac{t-t_{0}}{\hbar} E^{W}\left(p_{R}^{ \pm 0}\right)\right]}{E^{W}\left(p_{R}^{ \pm 0}\right)}\right\} \exp \left\{-\frac{i}{\hbar} p_{R}^{ \pm 0}\left(x-x_{0}\right)_{i} \sigma^{i}\right\}\left(\psi_{R}^{ \pm}\right)^{W}\left(x_{0}, t_{0}\right)+(1$ $+i \mu_{L}^{ \pm} c^{2} \frac{\sin \left[\frac{t-t_{0}}{\hbar} E^{W}\left(p_{L}^{ \pm 0}\right)\right]}{E^{W}\left(p_{L}^{ \pm 0}\right)} \exp \left\{-\frac{i}{\hbar} p_{L}^{ \pm 0}\left(x-x_{0}\right)_{i} \sigma^{i}\right\}\left(\psi_{L}^{ \pm}\right)^{W}\left(x_{0}, t_{0}\right)$,

And

*Corresponding author: Lukasz Andrzej Glinka, Member of the American Association of International Researchers, New York, USA, Tel: +48-660-019-237; E-mail: laglinka@gmail.com

Received February 11, 2014; Accepted February 11, 2014; Published February 18, 2014

Citation: Glinka LA (2014) On Neutrino in Deformed Relativity. J Astrophys Aerospace Technol 2: e107. doi:10.4172/2329-6542.1000e107

Copyright: @ 2014 Glinka LA. This is an open-access article distributed under the terms of the Creative Commons Attribution License, which permits unrestricted use, distribution, and reproduction in any medium, provided the original author and source are credited. 


$$
\begin{aligned}
& \left(\psi_{L}^{ \pm}\right)^{W}(x, t)=\left\{\cos \left[\frac{t-t_{0}}{\hbar} E^{W}\left(p_{L}^{ \pm 0}\right)\right]-\right. \\
& \left.+i p_{L}^{ \pm 0} c \frac{\sin \left[\frac{t-t_{0}}{\hbar} E^{W}\left(p_{L}^{ \pm 0}\right)\right]}{E^{W}\left(p_{L}^{ \pm 0}\right)}\right\} \exp \left\{-\frac{i}{\hbar} p_{L}^{ \pm 0}\left(x-x_{0}\right)_{i} \sigma^{i}\right\}\left(\psi_{L}^{ \pm}\right)^{W}\left(x_{0}, t_{0}\right)+ \\
& +i \mu_{R}^{ \pm} c^{2} \frac{\sin \left[\frac{t-t_{0}}{\hbar} E^{W}\left(p_{R}^{ \pm 0}\right)\right]}{E^{W}\left(p_{R}^{ \pm 0}\right)} \exp \left\{-\frac{i}{\hbar} p_{R}^{ \pm 0}\left(x-x_{0}\right)_{i} \sigma^{i}\right\}\left(\psi_{R}^{ \pm}\right)^{W}\left(x_{0}, t_{0}\right) .
\end{aligned}
$$

Where $E^{W}\left(p_{R}^{ \pm 0}\right) \equiv c^{2} \sqrt{\left(\sqrt{\mu_{R}^{ \pm} \mu_{L}^{ \pm}}\right)^{2}+\left(\frac{p_{R}^{ \pm 0}}{c}\right)^{2}}$.

In this manner, throughout the massive Weyl equation and manifestly unitary two-component Schrodinger equations, the model of Deformed Relativity leads to a massive normalizable neutrino states.

\section{References}

1. Glinka LA (2012) Aethereal Multiverse: A New Unifying Theoretical Approach to Cosmology, Particle Physics, and Quantum Gravity. Great Abington, UK. 